\title{
Patellar Tuberculosis presenting as an Osteolytic Lesion
}

\section{Devendra K Chouhan, Mandeep S Dhillon, Mahesh Prakash, Siddhartha Sharma}

\begin{abstract}
Tuberculosis of the patella is a rare entity, and may present with nonspecific clinical findings and subtle changes on plain radiographs; the rarity and unusual nature of presentation lead to frequent delays in diagnosis. We present the radiological (roentgenograms, CT scan and MRI) findings of a case of patellar tuberculosis and discuss the pertinent radiological parameters.
\end{abstract}

Keywords: Tuberculosis, Patella, Lytic lesion.

How to cite this article: Chouhan DK, Dhillon MS, Prakash M, Sharma S. Patellar Tuberculosis Presenting as an Osteolytic Lesion. J Postgrad Med Edu Res 2013;47(4):221-222.

\section{Source of support: Nil}

Conflict of interest: None declared

\section{INTRODUCTION}

A 28-year-old software engineer presented with insidious onset, moderately severe left knee pain (10 months) and insidious onset knee swelling (1 month); he had obtained several medical consults and taken over the counter analgesics without lasting relief. He stated that previous $\mathrm{X}$-rays were considered normal, and was being treated symptomatically. Physical examination at presentation revealed a swelling in the prepatellar region, increased local temperature, tenderness over the patella and normal range of motion of the knee joint. The hemoglobin was $10 \mathrm{gm} / \mathrm{dl}$, total leukocyte count was $11.5 \times 10^{9} \mathrm{gm} / \mathrm{l}$, erythrocyte sedimentation rate was $56 \mathrm{~mm} \mathrm{1st} \mathrm{hour} \mathrm{and} \mathrm{Mantoux} \mathrm{test}$ was $16 \mathrm{~mm}$.

X-rays of knee joint (AP and lat view) showed an ill defined lytic lesion in patella (Fig. 1). The lesion had geographical configuration with nonsclerotic margins and an ill-defined zone of transition (see Fig. 1). Magnetic resonace imaging (MRI) revealed a well defined focal lesion in the patella with linear fluid collection in prepatellar region and anterior to patellar tendon (Fig. 2). The lesion appeared hypointense on $\mathrm{T} 1$ and hyperintense on $\mathrm{T} 2 \mathrm{~W}$ images with a small breech anteriorly. Focal edema was seen in the rest of patella. Inflammatory stranding was noted in anterior fat pad. The other bones of knee joint were normal. No joint effusion was seen, and contrast study was not performed, so enhancement pattern could not assessed.

On basis of these findings, a presumptive diagnosis of a chronic infective etiology, probably tuberculosis, was made. The management options were discussed, and the patient was offered a biopsy to confirm the diagnosis; he chose to initiate antitubercular therapy (ATT) without a histo- pathological confirmation. The patient was started on a fourdrug daily regimen with isoniazid, rifampicin, pyrazinamide and ethambutol for 2 months after which three drug regime with isoniazid, rifampicin and pyrazinamide was continued for the next 4 months. Finally, isoniazid and rifampicin were given for 12 months. The patient had relief of pain and swelling after completing 1 month of ATT and no complications of ATT were noted. Post-treatment MRI showed decrease size of the patellar lesion with disappearance of prepatellar fluid (Fig. 3). Post-treatment CT scan showed a residual, well-defined lesion with nonsclerotic borders in patella with central sequestrum (Fig. 4). The cortical breech noted on pre-treatment MRI was not visualized and the adjacent soft tissue were normal.
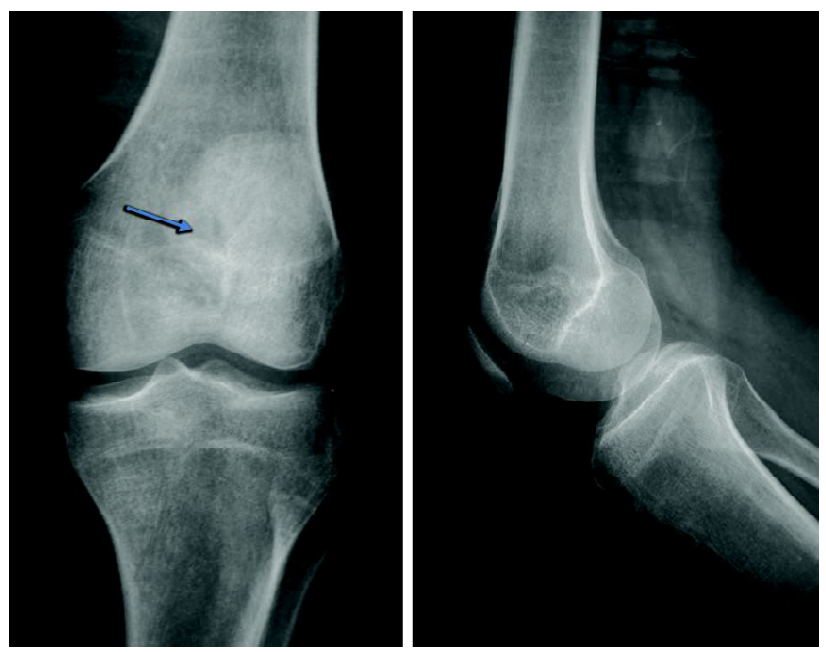

Fig. 1: X-rays of knee joint (AP and lat view) show ill-defined lytic lesion in patella (arrow)
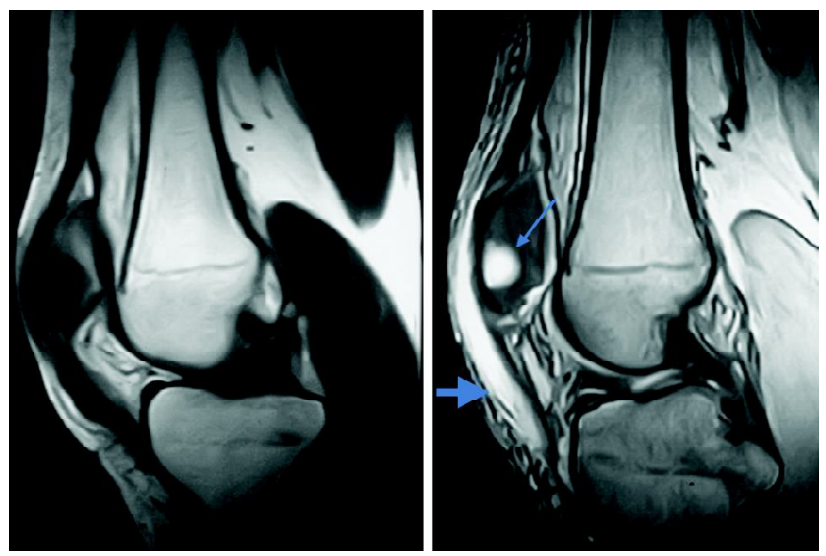

Fig. 2: T1 and T2W MRI sagittal images of the knee joint show well defined focal lesion (thin arrow) in the patella with linear fluid collection in prepatellar and anterior to patellar tendon (thick arrow) 

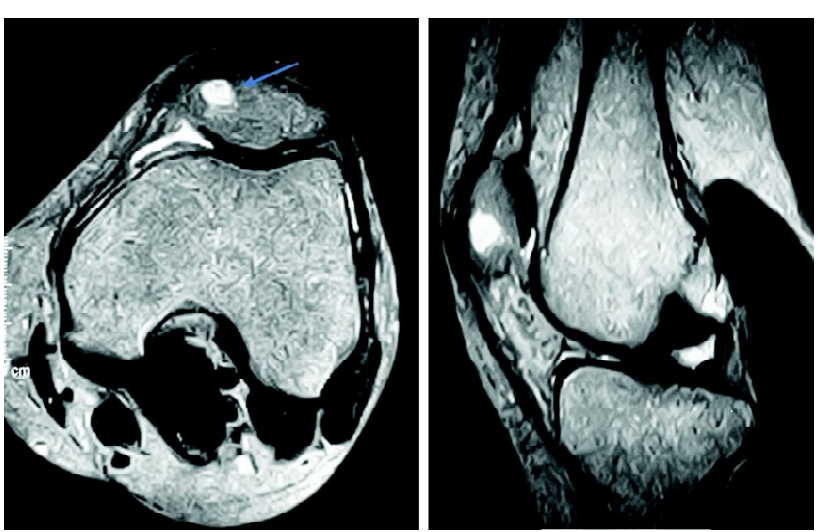

Fig. 3: Post-treatment MRI of the same patient (axial and sagittal T2W images) show decrease size of the patellar lesion with disappearance of prepatellar fluid
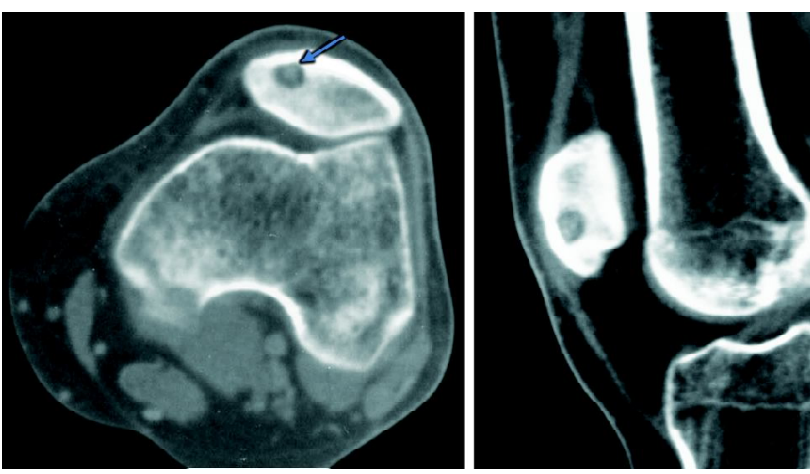

Fig. 4: Post-treatment CT scan (axial and sagittal reformatted images) show residual well-defined lesion in patella with central sequestrum

The first published account of patellar tuberculosis is that by Aitken et al who reported successful treatment by curettage. ${ }^{1}$ Tuberculosis of the patella is extremely rare and accounts for only $0.1 \%$ of all cases. ${ }^{2}$ Owing to its rarity, this condition is overlooked and often misdiagnosed. Since the pain and swelling are insidious in onset and the knee joint is spared, the patient often seeks medical attention late. Furthermore, the subtle radiographic findings may be easily missed on routine radiographs. A high index of suspicion is therefore needed on the part of the treating clinician. Though the AP and lateral radiographs of the patella are routine practice, skyline view of the patella skyline view of the patella is essential when we suspect any patellar lesions, and often delineates a focal defect very clearly. Computed tomography (CT) scan will readily show the osteolytic focus and define extent of sclerosis, as well as showing any sequestra. ${ }^{3,4}$ Furthermore, it can also show involvement of the patellofemoral joint, which is thought to be associated with poor outcomes. ${ }^{4} \mathrm{MRI}$ is helpful in evaluating the soft tissues around the knee, especially the prepatellar tissues which are characteristically involved in tuberculosis of the patella. ${ }^{5}$ Routine sequences for evaluating the patella are
T1W, T2W and STIR sequences in at least two planes. Contrast enhancement is required to know the enhancement pattern of the lesion and soft tissue collection. Tubercular osteomyelitis usually appears hypointense on T1 and hyperintense on $\mathrm{T} 2 \mathrm{~W}$ images with peripheral enhancement. Signal changes on MRI may persist for a long time, and the cavity may not be totally obliterated, so serial review is essential.

\section{CONCLUSION}

Patellar tuberculosis is a rare entity and must be considered in the differential diagnosis when a patient presents with insidious onset knee pain not responding to the usual line of management. The diagnosis may not be apparent on plain radiographs and a CT scan and/or MRI is needed to aid in the diagnosis. Diagnosis is usually confirmed on histopathology and a good outcome can be expected with antitubercular treatment. Nevertheless an indirect confirmation of diagnosis is by resolution of symptoms with a therapeutic trial of ATT.

\section{REFERENCES}

1. Aitken DM. Tuberculosis of the patella. Proc R Soc Med 1938 Jan;31(3):181.

2. Mittal R, Trikha V, Rastogi S. Tuberculosis of patella. Knee 2006 Jan;13(1):54-56.

3. Bonnet C, DeBandt M, Palazzo E, Malaizier D. Tuberculosis involving the patella. AJR Am J Roentgenol 1992 Sep;159(3):677.

4. Dhillon MS, Rao SS, Sandhu MS, Vasisht RK, Nagi ON. Tuberculosis of the patella. Skeletal Radiol 1998 Jan;27(1): 40-42.

5. Agrawal VK, Agrawal RG. Tuberculosis of patella in an immunocompetent patient - a case report. Indian J Tuberc Jan; 58(1):29-31.

\section{ABOUT THE AUTHORS}

\section{Devendra K Chouhan}

Assistant Professor, Department of Orthopedics, Postgraduate Institute of Medical Education and Research, Chandigarh, India

\section{Mandeep S Dhillon}

Professor, Department of Orthopedics, Postgraduate Institute of Medical Education and Research, Chandigarh, India

\section{Mahesh Prakash}

Associate Professor, Department of Radiodiagnosis, Postgraduate Institute of Medical Education and Research, Chandigarh, India

\section{Siddhartha Sharma (Corresponding Author)}

Senior Resident, Department of Orthopedics, Postgraduate Institute of Medical Education and Research, Chandigarh, India, Phone: 9988793537, e-mail: sids82@gmail.com 